Article

\title{
Microbiota Depletion Promotes Human Rotavirus Replication in an Adult Mouse Model
}

\author{
Roberto Gozalbo-Rovira ${ }^{1,+} \mathbb{D}^{\mathbb{D}}$, Cristina Santiso-Bellón ${ }^{1,+} \mathbb{D}^{\mathrm{D}}$, Javier Buesa ${ }^{1,2} \mathbb{D}^{\mathbb{D}}$, Antonio Rubio-del-Campo ${ }^{3}$, \\ Susana Vila-Vicent ${ }^{1}$, Carlos Muñoz ${ }^{1}$, María J. Yebra ${ }^{3}$, Vicente Monedero ${ }^{3, *}$ and Jesús Rodríguez-Díaz ${ }^{1,2, *(\mathbb{D})}$ \\ 1 Department of Microbiology, School of Medicine, University of Valencia, Av. Blasco Ibáñez 17, \\ 46010 Valencia, Spain; rovigoro@uv.es (R.G.-R.); cristina.santiso@uv.es (C.S.-B.); javier.buesa@uv.es (J.B.); \\ susana.vila@uv.es (S.V.-V.); carlos.munoz@uv.es (C.M.) \\ 2 Hospital Clínico Universitario de Valencia, Instituto de Investigación INCLIVA, 46010 Valencia, Spain \\ 3 Department of Biotechnology, IATA-CSIC, Av. Agustín Escardino 7, Paterna, 46980 Valencia, Spain; \\ anrucam@iata.csic.es (A.R.-d.-C.); yebra@iata.csic.es (M.J.Y.) \\ * Correspondence: btcmon@iata.csic.es (V.M.); jesus.rodriguez@uv.es (J.R.-D.); Tel.: +34-96-3983316 (V.M.); \\ +34-96-3900022 (ext. 2006) (J.R.-D.) \\ + These two authors contributed equally.
}

Citation: Gozalbo-Rovira, R.; Santiso-Bellón, C.; Buesa, J.; Rubio-del-Campo, A.; Vila-Vicent, S.; Muñoz, C.; Yebra, M.J.; Monedero, V.; Rodríguez-Díaz, J. Microbiota Depletion Promotes Human Rotavirus Replication in an Adult Mouse Model. Biomedicines 2021, 9 , 846. https://doi.org/10.3390/ biomedicines 9070846

Academic Editors: Federica Laudisi and Carmine Stolfi

Received: 23 June 2021

Accepted: 15 July 2021

Published: 20 July 2021

Publisher's Note: MDPI stays neutral with regard to jurisdictional claims in published maps and institutional affiliations.

Copyright: (c) 2021 by the authors. Licensee MDPI, Basel, Switzerland. This article is an open access article distributed under the terms and conditions of the Creative Commons Attribution (CC BY) license (https:// creativecommons.org/licenses/by/ $4.0 /)$.

\begin{abstract}
Intestinal microbiota-virus-host interaction has emerged as a key factor in mediating enteric virus pathogenicity. With the aim of analyzing whether human gut bacteria improve the inefficient replication of human rotavirus in mice, we performed fecal microbiota transplant (FMT) with healthy infants as donors in antibiotic-treated mice. We showed that a simple antibiotic treatment, irrespective of FMT, resulted in viral shedding for 6 days after challenge with the human rotavirus G1P[8] genotype Wa strain (RVwa). Rotavirus titers in feces were also significantly higher in antibiotictreated animals with or without FMT but they were decreased in animals subject to self-FMT, where a partial re-establishment of specific bacterial taxons was evidenced. Microbial composition analysis revealed profound changes in the intestinal microbiota of antibiotic-treated animals, whereas some bacterial groups, including members of Lactobacillus, Bilophila, Mucispirillum, and Oscillospira, recovered after self-FMT. In antibiotic-treated and FMT animals where the virus replicated more efficiently, differences were observed in gene expression of immune mediators, such as IL1 $\beta$ and CXCL15, as well as in the fucosyltransferase FUT2, responsible for H-type antigen synthesis in the small intestine. Collectively, our results suggest that antibiotic-induced microbiota depletion eradicates the microbial taxa that restrict human rotavirus infectivity in mice.
\end{abstract}

Keywords: rotavirus; antibiotic; microbiota; mice; virus shedding

\section{Introduction}

Diarrheal disease is the second leading cause of death worldwide in children under five years of age, accounting for around 525,000 deaths each year. Rotavirus (RV) is among the predominant causes of non-bacterial acute gastroenteritis in infant and young children, with an estimated 150,000 deaths per year, mostly in developing countries [1]. The gut is a very complex ecosystem, with multiple interactions between the host immune system, glycobiology, resident microbiota, and viruses responsible for gastroenteritis [2-5]. A link between human RV infection and intestinal bacterial populations has been revealed from analysis of the microbiota in population groups displaying different vaccine take after inoculation with RV live vaccines [6,7]. However, the role of commensal microbiota in RV infectivity is still controversial. Early evidences pointed towards a positive effect, showing reduced RV infectivity in germ-free or antibiotic-treated mice [8]. Contrarily, some microorganisms, including probiotic bacteria, directly interact with RV and potentially block their binding to epithelial receptors, counteracting infection [9]. 
RV attach to glycans of the histo-blood group antigen (HBGA) expressed in the gastrointestinal tract. In humans, fucosylation of O-linked HBGA at this location is controlled by FUT2 and FUT3 gene expression, and it has been demonstrated that the glycosylation patterns of those receptors could be altered by the action of specific commensal bacteria [10]. The main human RV genotype (G1P[8]) recognizes fucosylated HBGAs, such as H-type 1 [11], synthesized by FUT2 activity, and Lewis b antigens [12], synthesized by FUT3, but these viruses show inefficient spread or no replication at all in the mouse model [13].

With the aim of obtaining a versatile animal model for human RV infection, we tested whether engraftment of infant intestinal microbiota would permit G1P[8] RV infection in the mouse model. We showed that a simple microbial ablation through antibiotics was sufficient to promote human RV infection in mice, highlighting a role for intestinal microbiota in suppressing these RVs in the murine model.

\section{Materials and Methods}

\subsection{Rotavirus Stock Preparation}

A human RVwa strain was produced as previously described [14] with modifications. Briefly, 10 MA104-confluent $150 \mathrm{~cm}^{2}$ flasks (approximately $1.5 \times 10^{7}$ cells/flask) were infected with Wa RV at a multiplicity of infection (MOI) of 0.1 and the flasks were kept at $37^{\circ} \mathrm{C}$ for one week. After infection the virus was pelleted at $100,000 \times g$ for $2 \mathrm{~h}$ in a Himac R25ST-0507 rotor coupled to a Himac CR-30N $\times$ centrifuge. The pelleted virus was resuspended in TNC (20 mM Tris- $\mathrm{HCl} \mathrm{pH} 8.0,100 \mathrm{mM} \mathrm{NaCl}, 1 \mathrm{mM} \mathrm{CaCl}$ ) and ultra-centrifuged in a sucrose gradient (30-70\%) in TNC. The gradient was run in a SW41 rotor coupled to a Beckman L80 ultracentrifuge at $150,000 \times g$ for two hours. The band containing RV was collected and the virus was finally recovered by pelleting in the SW41 rotor coupled to the Beckman L80 ultracentrifuge for $2 \mathrm{~h}$ at 150,000 $\times g$ and resuspended in TNC. After preparation the RV Wa stock was titrated by qPCR, as previously described [15].

\subsection{Donor Microbiota Preparation}

Stool samples from four healthy human infants between one and three months of age were collected and resuspended in a solution consisting of $80 \% \times$-concentrated brain-heart infusion (Pronadisa, Madrid, Spain), supplemented with $0.1 \%$ cysteine, and $20 \%$ of a $200 \mathrm{~g} / \mathrm{L}$ skim milk (Scharlab, Barcelona, Spain) solution. This mixture was then diluted 1:2 $(v / v)$ in the same medium and stored in aliquots at $-80^{\circ} \mathrm{C}$. Mice fecal pellets from the animal groups were collected before the experiments and preserved using the same procedure.

\subsection{Antibiotic Treatment and Microbiota Stool Transplant}

Four groups of five C57BL/6J female 6 week-old mice were used in the present study. Three groups were treated with an antibiotic cocktail composed of $1 \mathrm{~g} / \mathrm{L}$ ampicillin, $1 \mathrm{~g} / \mathrm{L}$ metronidazole, $1 \mathrm{~g} / \mathrm{L}$ neomycin, and $0.5 \mathrm{~g} / \mathrm{L}$ vancomycin in drinking water, as previous described with modifications [16]. To diminish intestinal microbiota load, the animals were given the antibiotic-containing water ad libitum for three weeks and the antibiotic cocktail was renewed every three days. To determine the number of viable bacteria in mice stools, samples were collected at the beginning of the experiment and the day before RV infection, and serially diluted in brain heart infusion supplemented with $0.1 \%$ cysteine. The dilutions were plated in Wilkins-Chalgren medium containing $1.5 \%$ agar and incubated in anaerobic conditions (AnaeroGen, Sigma, Madrid, Spain) at $37^{\circ} \mathrm{C}$ for $48 \mathrm{~h}$. Mice were fed a standard diet until one week before fecal transplantation, when it was substituted by purified-defined germ-free diet (AIN-93G, Envigo, Barcelona, Spain ). 
The control group was maintained without antibiotics. Twenty-four hours after antibiotic treatment completion, two groups of mice were subjected to fecal material transplantation (FMT), as previously described with modifications [17]. One group of mice was transplanted with the preserved microbiota from the same group of mice before antibiotic treatment ( $100 \mu \mathrm{L}$ of prepared fecal material per mice for three consecutive days through oral gavage). A second group of mice received a FMT using the same procedure with a pool of bacteria from infant feces $(100 \mu \mathrm{L}$ of a pooled mix of four healthy infants for three days through oral gavage).

\subsection{Rotavirus Challenge}

Six days after FMT the mice were orally inoculated with $1 \times 10^{10}$ genome copy equivalent (GCE) of RVwa in $100 \mu \mathrm{L}$ of TNC. After RV dosing, the antibiotic-treated group without FMT continued the drinking water antibiotic treatment, whereas in the FMT mice groups, antibiotics were omitted from water from the day before microbiota transplant. Stool samples were collected daily for 7 days and kept at $-20{ }^{\circ} \mathrm{C}$. The mice were euthanized at 7 days post-infection (dpi) and the small intestine was removed and stored in RNAlater (Sigma) at $-80^{\circ} \mathrm{C}$ for further analysis.

\subsection{Quantification of Rotavirus from Stool Samples by RT-qPCR}

We extracted RNA from mice stool samples using the NucleoSpin RNA Virus (MachereyNagel, Duren, Germany) kit following the manufacturer's instructions. The primers and probe sequences utilized for RT-qPCR have previously been described [15]. Amplification of RNA samples was performed with one-step TaqMan RT-qPCR using the RNA UltraSense One-Step quantitative system (Thermo Fisher Scientific, Madrid, Spain). The standard curve was generated by serial end-point dilution, amplifying 10-fold dilutions of the quantified plasmid containing the RV target sequence by RT-qPCR in triplicates.

\subsection{Quantification of Cytokine and Glycosyltransferase mRNA Expression Level}

Then, $100 \mathrm{mg}$ of tissue from the small intestine of infected mice were homogenized in $1 \mathrm{~mL}$ of Trizol (Thermo Fisher Scientific) using a Polytron PT10-35 GT (Thermo Fisher Scientific) at $16,000 \mathrm{rpm}$. After tissue disruption the RNA was purified following the manufacturer's instructions and the RNA finally resuspended in $50 \mu \mathrm{L}$ of DEPC-treated water. The RNA was treated with RNAse-free DNAse I (Thermo Fisher Scientific) to remove the contaminant DNA and retro-transcribed to cDNA using the SuperScript III enzyme (Thermo Fisher Scientific) and random primers following the manufacturer's instructions. The cDNA obtained was kept at $-20{ }^{\circ} \mathrm{C}$ until use. Expression levels of genes encoding cytokines IL1 $\beta, I L 4, I L 6, C X C L 15, I L 10, I L 12, I L 13, T N F \alpha, I F N \gamma$, and TLR2 were studied in a LightCycler480 Instrument SW1.5 (Roche Life Science, Basel, Switzerland) and the expression analysis performed with the Rest Software [18]. The expression level of GAPDH and RPLPO housekeeping genes was used as reference. A list of primers employed can be found in Supplementary Table S1.

Glycosyltransferase FUT2 gene expression was also analyzed by RT-qPCR. Primer and probe preparation for the gene was purchased from Integrated DNA Technologies, Inc. (IDT, assay ID: Mm.PT.58.50508299). The NZYSpeedy qPCR Probe Master Mix (NZY, Lisbon, Portugal) was utilized. The qPCR was run in the StepOnePlus Real-Time PCR System (Applied Biosystems) and expression analysis was performed with Rest Software [18], using HPRT gene expression (assay ID: Mm.PT.39a.22214828) as a reference.

\subsection{Microbiota Profiling in Mice Stool Samples}

Total rDNA $16 S$ in mice feces was quantified by qPCR as previously described [19]. To assess microbiota composition, DNA was extracted from mice fecal pellets obtained prior to RVwa inoculation from all experimental groups with the Master Pure ${ }^{\mathrm{TM}}$ kit (Epicentre), including a bead beater treatment with $0.5 \mathrm{~g}$ of $0.1 \mathrm{~mm}$ glass beads in the lysis step (FastPrep 24-5G Homogenizer; MP Biomedicals, CA, USA), followed by final purification of the 
extracted DNA samples with mi-PCR Purification kit (Metabion, Planegg-Steinkirchen, Germany). DNA was quantified with a Qubit 2.0 fluorometer (Invitrogen, Thermo Fisher Scientific, Madrid, Spain). Bar-coded amplicons of the $16 \mathrm{~S}$ rDNA V3-V4 region, multiplexed using Nextera XT Index Kit, were subject to $2 \times 300$ bp paired-end run in a MiSeq-Illumina platform (SCSIE, University of Valencia, Valencia, Spain). Data were demultiplexed using Illumina bcl2fastq@ program and reads were checked for quality, adapter trimmed and filtered using AfterQC and FastQC v0.11.8 (http:/ / www.bioinformatics.babraham.ac.uk, accessed 10 May 2021) tools. QIIME software V1.9.1 [20] was used to analyze MiSeq sequencing data, including forward and reverse reads joining, chimera removal, data filtering, and taxonomic annotation. Chimeric sequences were removed from the reads using the USEARCH 6.1 algorithm. Reads were clustered into operational taxonomic units (OTUs) based on a 97\% identity threshold value. Sequence were aligned to the Greengenes core reference database (version 13.8) using PyNAST. Taxonomic assignment was made using the UCLUST classifier. A total of 4,356,240 non-chimeric reads were obtained, with a mean of 207,440 sequences per sample. Datasets were rarefied to the minimum library size $(43,791$ reads) and normalized by total-sum scaling prior analysis with Calypso 8.84 (http:/ / cgenome.net/calypso/ accessed 10 May 2021) and MicrobiomeAnalyst (https: / / www.microbiomeanalyst.ca/ accessed 10 May 2021; [21] software pipelines.

\section{Results}

3.1. Mice with Depleted Microbiota and Transplanted with Human Feces Shed Wa Rotavirus for 6 Days

We reasoned that in the intestinal niche of infants where RV develop, the resident microbiota determines infectivity. Then, we asked whether infection of RVwa (G1P[8]) in the mouse model could be improved by replacing its intestinal microbiota with the microbiota of infants by FMT using antibiotic (Ab)-mediated microbiota ablation. In order to answer this, C57BL/6J mice were treated with an antibiotic cocktail through drinking water for three weeks. Bacterial counts in fecal pellets decreased from $6.3-9.7 \times 10^{10} \mathrm{CFU} / \mathrm{g}$ to no detectable counts (plating $10^{-1}$ dilutions), whereas they remained stable in the nontreated control $\left(4.9 \times 10^{10} \mathrm{CFU} / \mathrm{g}\right)$, indicating an efficient depletion of the culturable gut bacteria. Mice were then subject to FMT and orally dosed with the Wa strain. The virus could be detected in the feces of control animals with a peak at one day post-infection (dpi; $9.1 \times 10^{9}$ genome copy equivalent (GCE) $/ \mathrm{g}$ feces), which rapidly dropped, with RV being under the detection limit at dpi 4 (Figure 1). By the contrary, compared to control animals, at dpi 1 viral shedding in Ab-treated mice and in mice subject to human FMT (hFMT) was 23- and 16-fold higher $\left(2.1 \times 10^{11} \mathrm{GCE} / \mathrm{g}\right.$ and $1.5 \times 10^{11} \mathrm{GCE} / \mathrm{g}$, respectively) and remained until dpi 5-6, following the same evolution pattern and with values higher than $10^{9} \mathrm{GCE} / \mathrm{g}$. The lower levels of RV detected in control animals and its faster clearance were indicative of an inefficient replication, in contrast to the prolonged viral shedding in $\mathrm{Ab}$-treated mice and in the hFMT group. In mice receiving a self-FMT, partial restoration of the restrictive replication of RVwa was evidenced, and virus shedding was detected at levels similar to control at dpi 1, with no detectable viral shedding at dpi 4 (Figure 1).

\subsection{Impact of Fecal Microbiota Transplant on Mouse Microbiota}

The results of viral shedding pointed to microbiota changes by Ab treatment and hFMT as responsible for RVwa infection permissiveness in mice. The treatment with antibiotics significantly reduced the number of bacteria calculated by 165 rDNA qPCR (Figure 2). Both the transplant from human feces and the self-transplant increased the number of bacteria with no significant differences between them (Figure 2). 


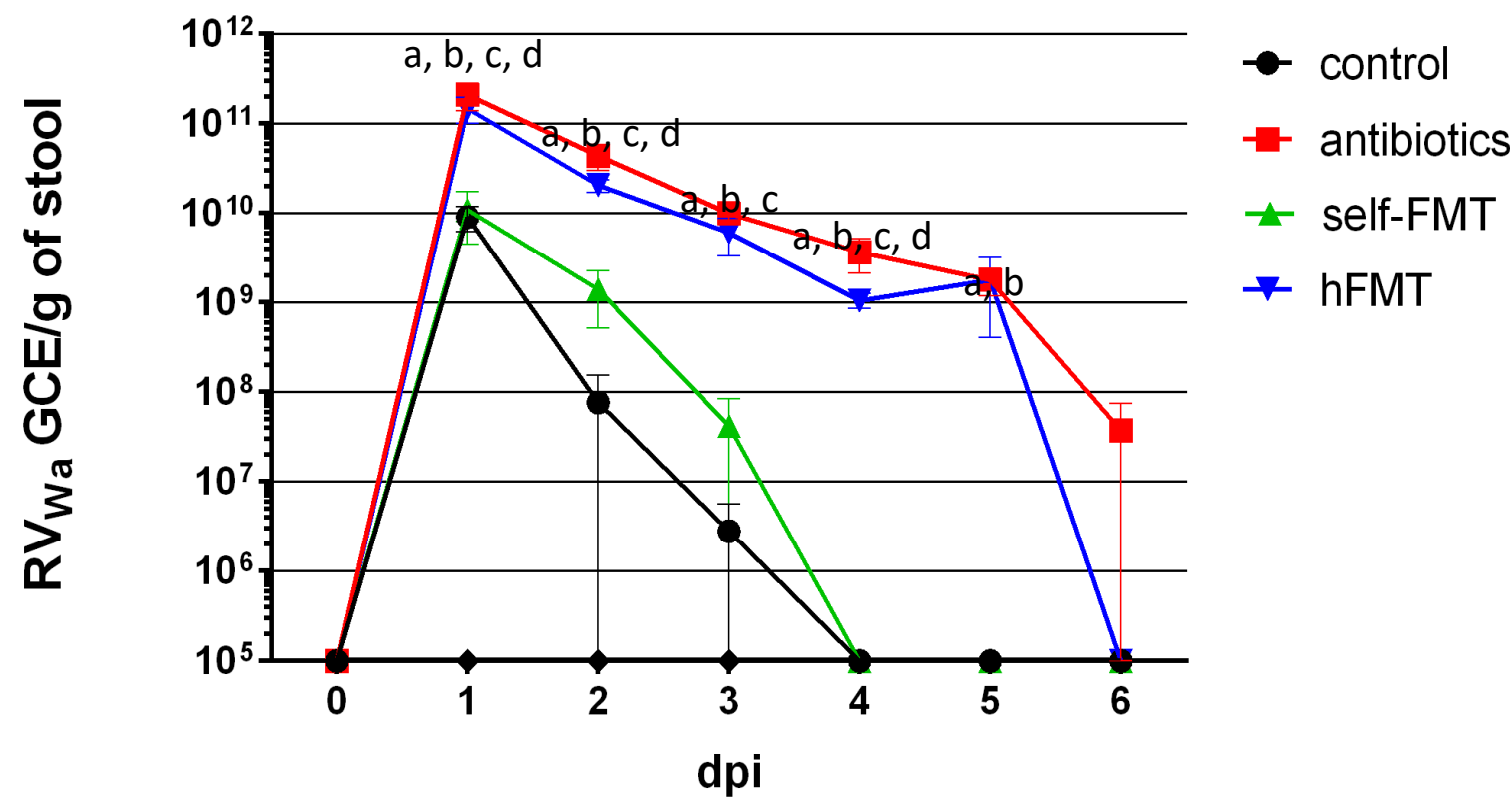

Figure 1. Rotavirus Wa shedding in mice stool. GCE/g of stool are presented for control mice, Ab-treated mice and mice subject to hFMT and self-FMT, respectively. The mice were followed for 6 days and euthanized on day 7. The letters indicate statistically significant differences $(p<0.05)$ in the GCE between the groups $(\mathrm{a}, \mathrm{Ab}$ vs. control; $\mathrm{b}, \mathrm{Ab}$ vs. self-FMT; c, hFMT vs. control; $d$, hFMT vs. self-FMT). The error bars are standard deviations $(n=5)$. The limit of detection was $10^{5} \mathrm{GCE} / \mathrm{g}$ of stool. Ab, antibiotic; hFMT, human fecal microbiota transplantation; self-FMT, self-fecal microbiota transplantation.

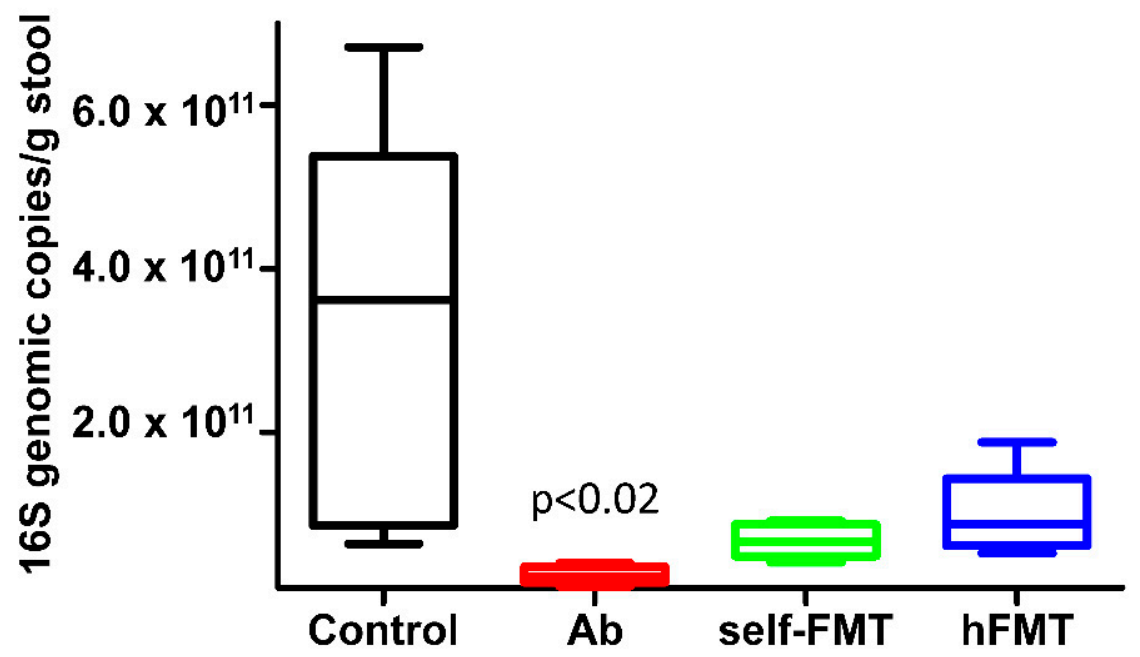

Figure 2. Total bacteria 16S DNA quantification by qPCR. The 16S DNA genomic copies/g of stool are presented for control mice, Ab-treated mice and mice subject to self-FMT and hFMT, respectively. Ab-treated mice had significantly lower amount of genome copies than the other three groups $(p<0.02)$. No significant differences were found between the human and self-transplanted groups.

The microbiota composition in feces prior to RV challenge was determined by $16 \mathrm{~S}$ rDNA NGS, showing that bacterial richness and diversity was severely affected after the different treatments (Figure 3A). Chao1 index was lowered in all groups compared to control (microbial richness; $p=2.0365 \times 10^{-12} ; \mathrm{F}=206.49$, ANOVA) but was higher in self-FMT animals. Diversity (Shannon index) was also lower $\left(p=1.8988 \times 10^{-8} ; \mathrm{F}=57.299\right.$, ANOVA), but was higher in the hFMT group compared to self-FMT group, indicating uneven microbial distribution after treatments. In both types of analyses $\mathrm{Ab}$ treatment resulted in lower $\alpha$ diversity. As expected, the three distinct treatments caused profound 
remodeling of the intestinal microbial profile and all groups could be differentiated in terms of overall composition (Figure 3B,C). In mice treated with Ab, members of the phyla Firmicutes and Proteobacteria, belonging to the genus Lactococcus and unidentified $\gamma$-Proteobacteria (enterobacteria), respectively, accounted for most of the microbiota (Figure 3B).
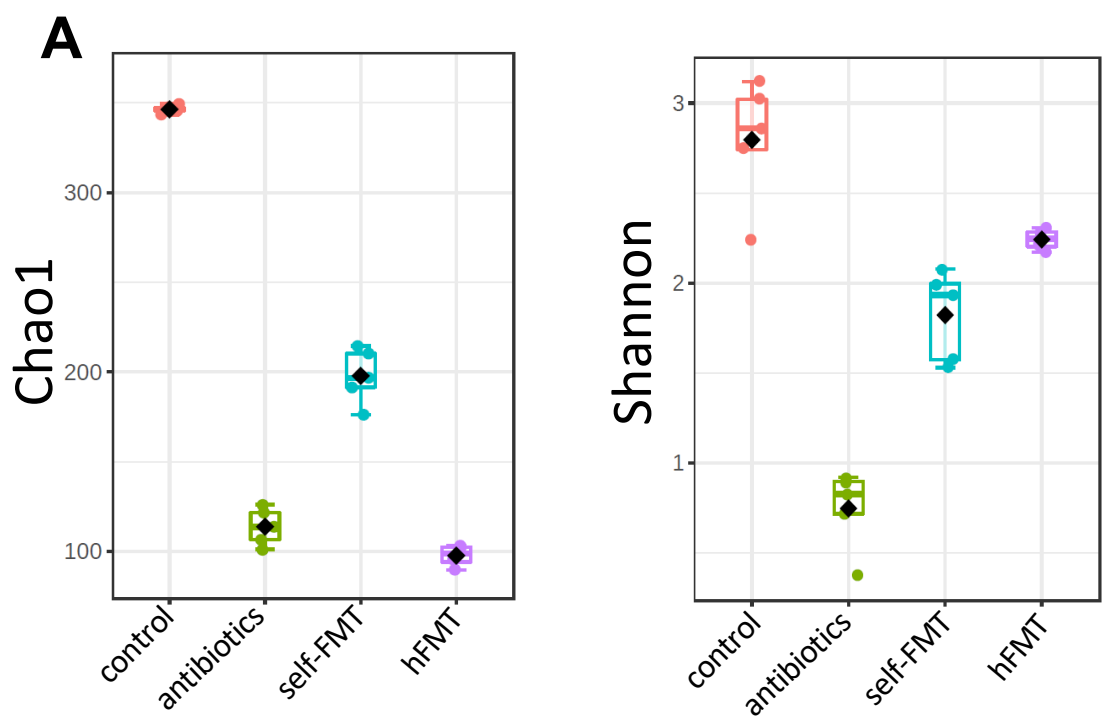

B
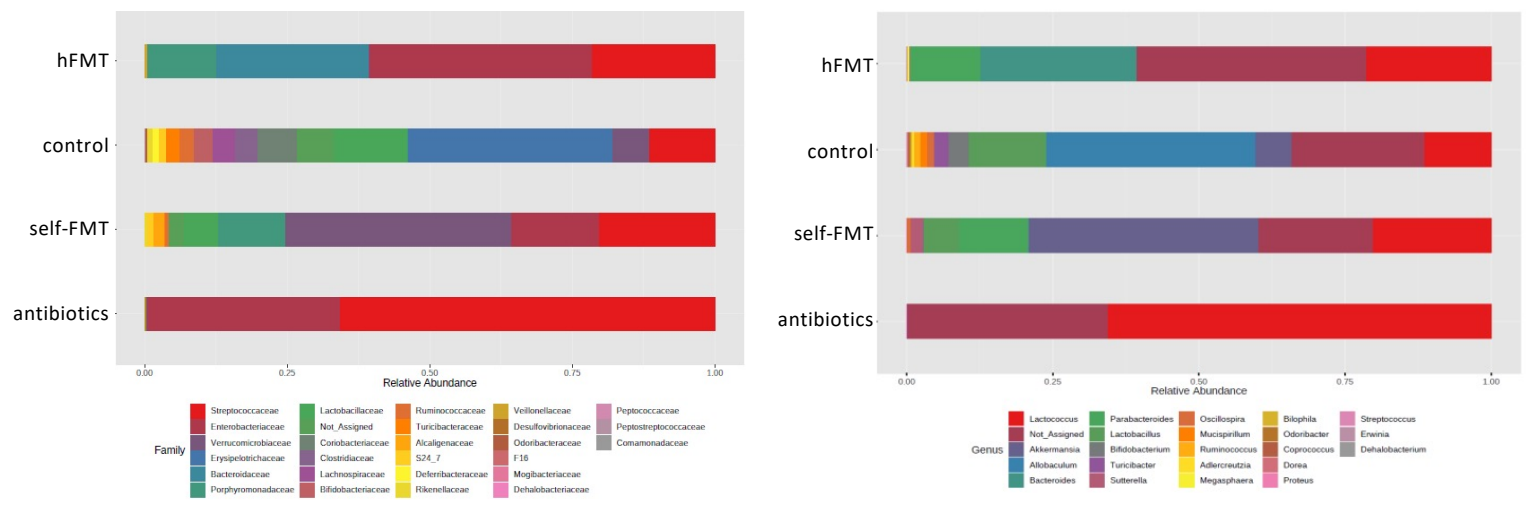

C

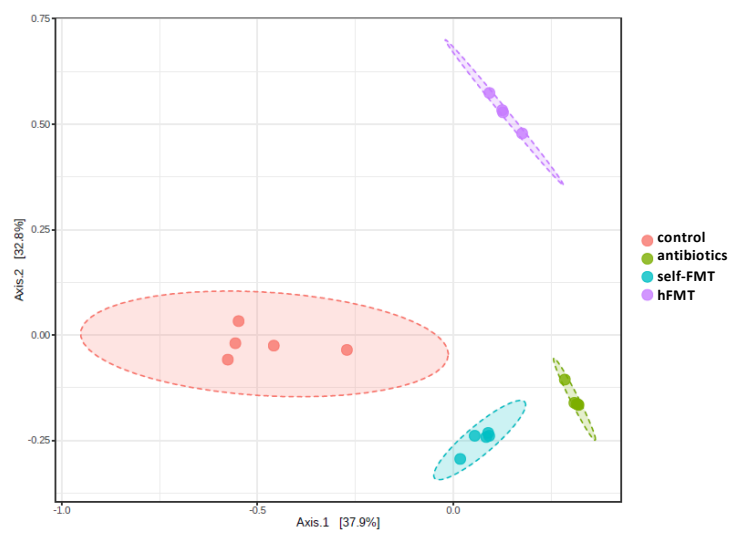

Figure 3. Changes in intestinal bacterial composition in the mice groups. (A) Microbial $\alpha$-diversity. Microbial richness (Chao1) and diversity (Shannon) indexes are shown. (B) Relative abundance of bacterial taxa (family and genus levels) found in the feces of the different mice groups. (C) Differences in microbial global composition after the different treatments. A principal coordinate analysis (PCoA) of the Bray-Curtis dissimilarity indexes of samples is shown (PERMANOVA F-value $\left.=36.77 ; R^{2}=0.8803 ; p<0.001\right)$. 
The microbiota present in the pooled infant feces was not completely engrafted in mice after hFMT (Figure 4), but these mice were characterized by elevated numbers of Bacteroidetes and presence of members of the genus Megasphaera, a bacterial taxon that was absent in the microbiota of untreated mice (Figure 5A). Genera, such as Bifidobacterium, Adlercreutzia, Ruminococcus, Coprococcus, Turicibacter, Odoribacter, and Allobaculum, were completely depleted after $\mathrm{Ab}$ treatment and they were not replenished by any FMT, including self-FMT (Figure 5B). Although microbial engraftment was not completely successful in the self-FMT group (differences in $\alpha$ and $\beta$ diversity were obvious compared to control animals), bacteria belonging to Oscillospira, Lactobacillus, Mucispirillum, and Bilophila were partially restored in mice via self-FMT (Figure 5C). In addition, in self-FMT animals proportions of Akkermansia and Sutterella taxons were increased compared to control mice (Figure 5D). In summary, low microbial richness and ablation of most representative taxa of mice microbiota was concomitant with higher mice permissiveness to RVwa infection.

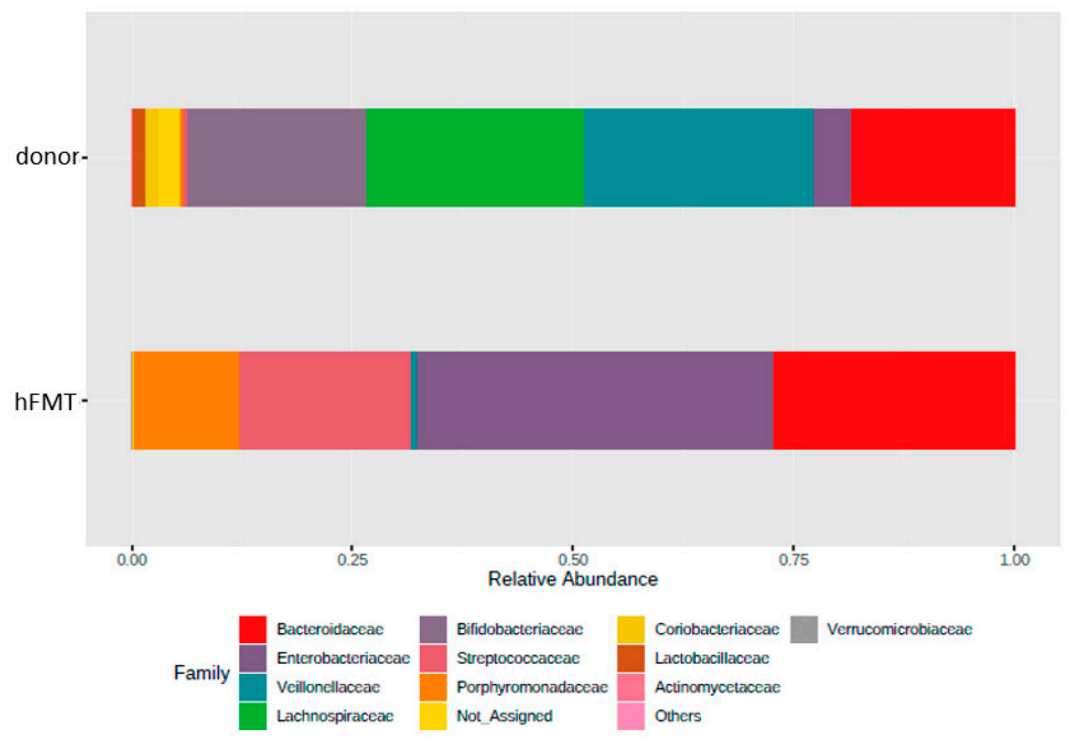

(A)

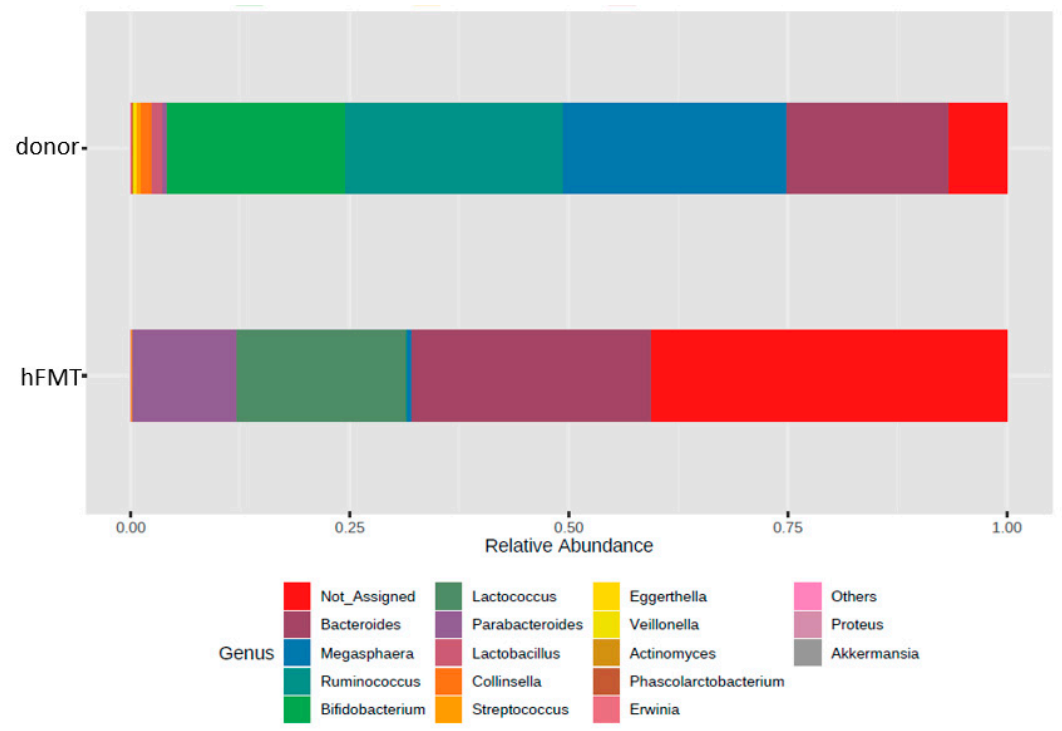

(B)

Figure 4. Relative abundances of bacterial taxa (family and genus) in donor feces and feces of mice subject to hFMT. The panel (A) shows the relative abundance of bacteria at family level of the donor sample while panel (B) shows the same at genus level. 
A

B
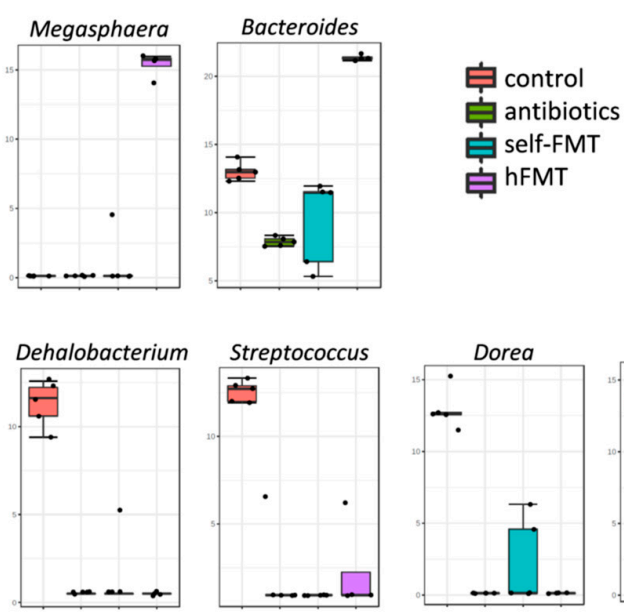

Coprococcus

Odoribacter

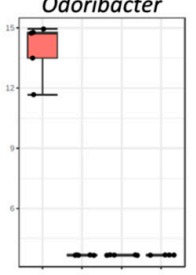

Adlercreutzia

Ruminococcus
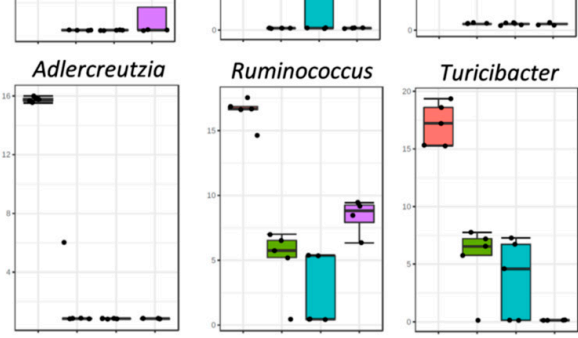

Bifidobacterium

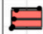

Allobaculum

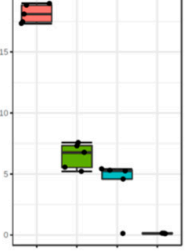

콛

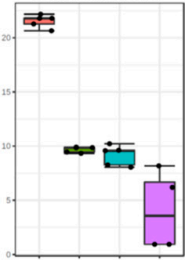

C
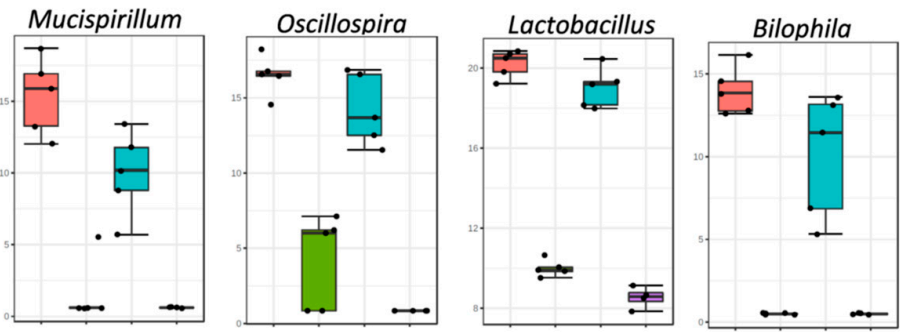

D
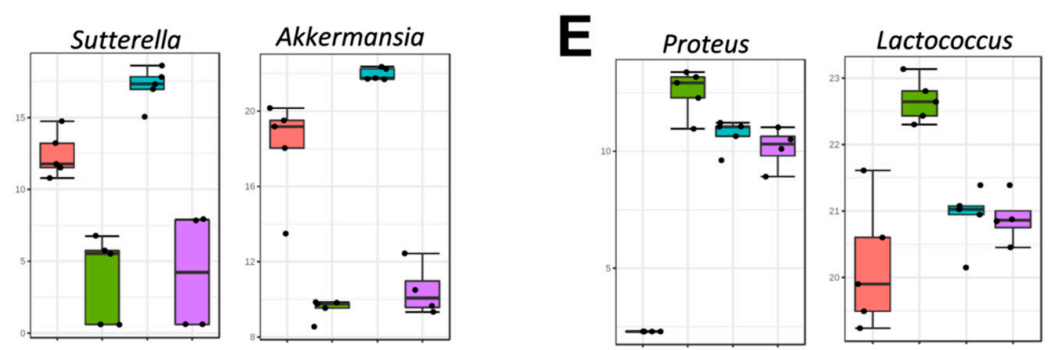

Figure 5. Bacterial genera showing differences between treatment groups. A LEfSe analysis was performed to detect bacterial markers characteristic of each treatment condition. The different box-plots represent abundance (log transformation of data normalized by total-sum scaling $\times 10^{7}$ ) of bacterial genera showing LDA scores $>4$. (A) Bacteria augmented after hFMT; (B) Genera ablated or diminished by Ab treatment; (C) Bacteria that were partially restored by self-FMT; (D) Bacteria augmented after self-FMT; (E). Bacteria increased in Ab-group. Red, control animals; green, antibiotics-treated animals; blue, self-FMT; magenta, hFMT. 


\subsection{Immune and Epithelial Glycosylation Gene Expression in the Gut Associates with Human Rotavirus Infection in Mice}

Gene expression levels of a panel of cytokines and other innate immune system mediators including IL1 $\beta$, IL4, IL6, CXCL15, IL10, IL12, IL13, IFN $\gamma$, TNF $\alpha$, and TLR2 were studied by RT-qPCR in the small intestine of mice at 7 dpi (Figure 6). IL6, IL12, and IL13 expression levels did not differ between experimental groups and control animals, whereas a downregulation effect was generally observed in the remaining tested genes. IL1 $\beta$ and CXCL15 messenger levels were lower in the Ab and hFMT groups where human rotavirus replicated more efficiently. Expression of IL10, TLR2, and TNF $\alpha$ was also reduced in all groups compared to control. Finally, IFN $\gamma$ expression was diminished in mice subject to both FMT treatments. Expression of the FUT2 gene, whose product is involved in $\alpha 1,2$-fucosylation during the synthesis of mucosal $\mathrm{H}$ type- 1 antigen (fucosyl- $\alpha 1$,2-galactopyranosyl- $\beta 1,3-$ $\mathrm{N}$-acetyl-glucopyranoside), one of the P[8] RV adhesin receptors, was downregulated in self-FMT animals. Interestingly, the FUT2 gene expression differed between both transplanted groups, having a higher expression $(p=0.003)$ in the group that received the human feces transplant (Figure 6). More interestingly, a significant negative correlation was found between the expression level of IL1 $\beta$ and the rotavirus shedding in feces at 2 dpi (Pearson $\mathrm{r}=-0.7203, p=0.0005)$. More moderate correlations were also found with TLR2 (Pearson $\mathrm{r}=-0.5824, p=0.0089$ ) and TNF $\alpha$ (Pearson $\mathrm{r}=-0.4654, p=0.0446)$.
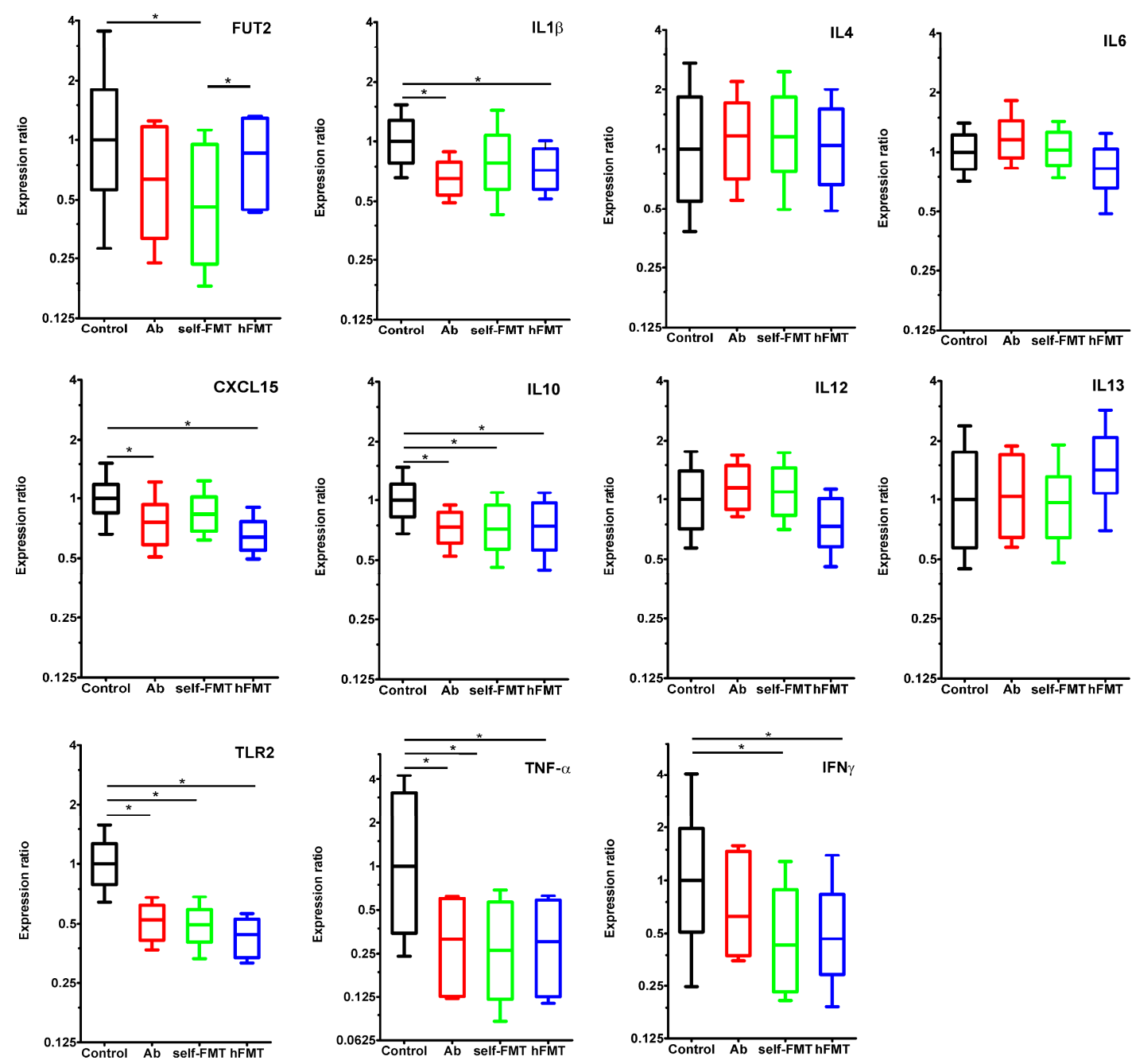

Figure 6. Differences in gene expression in the small intestine of infected mice. Expression of immune system mediators (IL1 $\beta, I L 4, I L 6, C X C L 15, I L 10, I L 12, I L 13, I F N \gamma, T N F \alpha$, and TLR2) and fucosyltransferase 2 (FUT2) was determined by RT-qPCR in mice from the different groups at $7 \mathrm{dpi}(n=5)$. The level of significance is indicated for control mice $\left({ }^{*} p<0.05\right)$. 


\section{Discussion}

Gut microbiota has emerged as a pivotal player in enteric virus-host interactions, and has been shown or hypothesized to have positive, as well as negative effects on viral infectivity, mediated by different mechanisms [2-5]. Given the lack of an efficient mouse model to replicate relevant human RV strains [13], we studied whether mice could be infected with RVwa after an infant gut microbiota engraftment. This RV strain has been used as a model for human RV studies and has special relevance because it carries the $P[8]$ VP4 genotype, which is responsible for around $90 \%$ of infections [22]. An available in vivo model of replication would therefore be desirable, yet its infectivity in small animal models is not satisfactory.

In our study, RVwa infection could not be linked to hFMT, ruling out the possibility that human intestinal microbiota mediates RVwa infectivity in mice. Nonetheless, we show that independently of subsequent hFMT, simple gut bacteria depletion through antibiotic treatment dramatically increased the replication capacity of the RVwa strain in this host. Altered intestinal bacterial loads in the mice do not account for the differences in RVwa replication, as no differences were found in bacterial numbers in the feces from groups that substantially differed in RVwa infection. It has been discussed that variations in the microbiome of experimental animals at different laboratories may affect mice phenotypes, which can be at the basis of reproducibility issues [23]. This situation would have minor effects in our case, as we showed that the sole fact of depleting the microbiota with antibiotics allowed RVwa replication. Our data suggest that endogenous mouse microbiota restricts RVwa replication, which is in opposition to results obtained with the murine RV $\mathrm{EC}$ strain, where $\mathrm{Ab}$ treatment had negative effects on viral entry, delaying and decreasing infectivity in both adult (40\% reduction in viral shedding) and neonatal (34\% reduction in diarrhea incidence) murine models [8]. However, recent research also points to a protective role for the microbiota during murine RV (strain EDIM) infection, by demonstrating high susceptibility to RV infection in Ab-treated and germ-free animals and highlighting a role of microbiota-induced IL22 expression as an anti-viral mediator [24].

The importance of the microbiota in establishing an appropriate anti-viral immune response has been noted $[25,26]$. Exacerbated systemic infection by several enteric and non-enteric viruses was observed in Ab-treated mice, including vesicular stomatitis and influenza virus [27], murine gamma herpesvirus [28], respiratory syncytial virus [29], encephalomyocarditis virus [30], and West Nile, Dengue, and Zika viruses [31]. In some cases, microbiota depletion has been linked to a defective innate immune response characterized by low levels of type I IFN expression (IFN $\beta$ ), which hampered the ability to mount an effective anti-viral macrophage response [27,29]. In our experiments, antibiotic treatment generally resulted in reduced expression of inflammatory mediators in the small intestine, although no IFN $\beta$ expression could be detected in the whole tissue (data not shown). We found that IL10 and TNF $\alpha$ displayed reduced expression in microbiota-depleted animals and that IFN $\gamma$ expression was also lower in FMT animals. These are important mediators of immune response to infections, including RV, in which studies have previously reported increased expression after infection in a mouse model [32,33]. Accordingly, although germfree mice show delayed infection with EC RV, viral clearance in this model was found to last substantially longer, probably reflecting an immature intestinal immune system [8]. IL1 $\beta$ expression was lowered in Ab and hFMT groups and negatively correlated with viral shedding. This cytokine plays a major role in early inflammatory response against pathogens and its level is increased during RV infection in children [34]. Therefore, reduced IL10, IL1 $\beta, T N F \alpha$, and IFN $\gamma$ are likely to be beneficial for viral replication. TLR2, whose expression was lowered in the Ab-treated groups, has been linked to the protective effect of lactobacilli towards RV infection in animal models [35,36]. We also studied the association between RV infection and FUT2. It was previously shown that specific members of the gut microbiota, such as Bacteroides thetaiotaomicron, Bacteroides fragilis, and segmented filamentous bacteria (SFB) are able to influence the pattern of mucosal glycosylation by inducing epithelial FUT2 expression [10,37], which encodes the enzyme responsible for 
H-type antigen synthesis, the target for binding of RV P[8] adhesin [11]. We showed that FUT2 expression in the small intestine was only reduced in self-FMT animals. Lack of intestinal microbiota generally results in downregulation of FUT2 [37], and it is not known how diminished FUT2 levels could affect RVwa infection in mice, especially given that another entry mediator of P[8] RV, the Lewis b antigen [12], is not produced in this host. A recent report shows that virulent RVwa (a strain serially passed in gnotobiotic pigs) infection in porcine enteroids is enhanced by the presence of $\mathrm{H}$ antigen [38], but the role of HBGA in infection in mice has yet to be determined.

The limited RVwa infection found in self-FMT animals suggests that specific microbial taxa not implanted after hFMT are implicated in RV exclusion. Although self-FMT did not restore the original microbiota of mice, several bacterial taxa with a potential role in RVwa restriction were partially restored: Mucispirillum is a strict anaerobe typically found in the murine intestinal tract, where it is intimately linked to the mucosal layer and participates in inflammatory processes [39] and is also involved in excluding several pathogenic bacteria [40]; Oscillospira is another anaerobe from the grastrointestinal tract, and, although it has never been cultivated, evidence indicates its relevance to human health [41]; Bilophila, which comprises the single species Biliophila wadsworthia, has been linked to intestinal inflammation in mice [42]; and finally, lactobacilli are well-known probiotic bacteria that have been shown to diminish RV-induced diarrhea in humans [43] and reduce RV infection in both in vitro and in vivo models [44,45].

A recent study showed that members of the murine intestinal microbiota such as SFB belonging to Candidatus Arthromitus are responsible for inhibiting murine RV EC strain infection [46]. Thus, Ab elimination of SFB could explain Ab-treated mice tolerance to RVwa infection. However, SFB were not found in our microbiota analysis and the model where they were implicated in resistance to the murine RV strain EC used immunosuppressed Rag1-KO mice, which are defective in B and T lymphocytes. However, Candidatus Arthromitus was not able to stably colonize normal mice [46]. Nevertheless, the idea that some bacterial taxa, such as those re-established after self-FMT or others, could mediate RVwa exclusion in mice is still attractive. Such a category of bacteria has been identified and the anti-viral mechanisms behind them disclosed in some cases. As examples, oral dosing of Blautia coccoides in Ab-mice restored the capacity of macrophages to induce IFN $\beta$ and promoted protection against encephalomyocarditis virus systemic infection [30], and, likewise, lipo-oligosaccharides from the outer membrane of Bacteroides and microbial metabolism-derived acetate were involved in triggering an IFN $\beta$ response that prevented vesicular stomatitis virus and influenza virus [27] and respiratory syncytial virus [29] infection in mice, respectively.

RVwa permissiveness in Ab-treated mice could also derive from depletion of one or various of the many microbial taxa affected by this treatment but not restored by selfFMT. As an example, Ruminococcus practically disappeared after Ab administration. These bacteria were correlated with low anti-RV IgA titers in human saliva of healthy subjects [47], have recently been characterized as microorganisms physically interacting with RV in the stools of children suffering P[8] RV-induced diarrhea, and reduced RVwa infectivity in Caco-2 cell cultures [9]. Bifidobacterium, another relevant genus of probiotic bacteria with proven anti-RV properties $[45,48]$ was also eradicated by antibiotics.

Alternative mechanisms by which bacteria could mediate RV protection include direct interaction of RV virions with bacteria, as hypothesized for SFB [46]. These interactions have been evidenced in in vitro experiments for a number of bacterial strains [32,49]. Species such as Ruminococcus gauvreauii [9] and others [50] express HBGA-like substances on their surface which are binding targets for RV. HBGAs act as virion stabilizers that promote bacteria-assisted binding and enhance infection in some enteric viruses, such as norovirus (NoV) and poliovirus [51-53] but can mediate virus sequestration on the bacterial surface, which could prevent virus from binding to target cells.

The mechanisms underlying RVwa development in antibiotic-treated mice are as yet unknown, but our results suggest either that natural mouse resistance to RVwa infection is 
triggered by indigenous mouse commensals that cannot be substituted by other human intestinal bacteria, or, alternatively, that the equivalent human microorganisms cannot be implanted in the mouse by simple FMT. Further characterization of the factors mediating RV replication in Ab-treated mice is needed. Nonetheless, this in vivo infection model may constitute a valuable tool to investigate the biology of RV. Identifying the bacteria responsible for human RV restriction in mice will further understanding of the relationship established between RV and intestinal commensals.

Supplementary Materials: The following are available online at https:/ /www.mdpi.com/article/10 $.3390 /$ biomedicines $9070846 /$ s1, Table S1: primers used for qPCR in the present study.

Author Contributions: Conceptualization, J.R.-D., J.B., V.M., R.G.-R., C.M., A.R.-d.-C., M.J.Y.; Methodology, R.G.-R., C.S.-B., A.R.-d.-C., C.M., S.V.-V., V.M.; Writing-Original Draft Preparation, J.R.-D., V.M.; Review and Editing, A.R.-d.-C., J.B., M.J.Y., C.M., R.G.-R., C.S.-B.; Project Administration J.R.-D.; Funding Acquisition, J.R.-D., M.J.Y. All authors have read and agreed to the published version of the manuscript.

Funding: This research was funded by Spanish Government (Ministerio de Economía y Competitividad) grants AGL2017-84165-C2-1-R to MJY, AGL2017-84165-C2-2-R to JRD. The study was also supported by Valencian Government grant IDIFEDER/2018/056 and by European FEDER founds. RGR is the recipient of a postdoctoral grant from the Valencian Government (APOST/2017/037). CSB is the recipient of a FPI predoctoral grant from the Spanish Government (RE2018-083315). SVV is the recipient of a predoctoral grant from the Valencian Government (ACIF/2016/437).

Institutional Review Board Statement: All experiments using mice were conducted at the Animal Production and Experimentation Service of the University of Valencia following national and international regulations. The procedures were approved by Ethics Committee for Animal Welfare and by the "Dirección General de Agricultura, Ganadería y Pesca" of the "Generalitat Valenciana", file number 2018/VSC/PEA/0181 approved the 19 December 2018. Use of human stool samples was approved by the Human Research Ethics Committee of the University of Valencia (registration number H154401046838) and written informed consent was obtained from a parent of each subject.

Informed Consent Statement: Informed consent was obtained from all subjects involved in the study.

Data Availability Statement: The raw sequencing fastq files were deposited in the SRA repository (http:/ / www.ncbi.nlm.nih.gov/sra accessed 1 July 2021) under Bioproject ID PRJNA706108.

Conflicts of Interest: The authors declare no conflict of interest.

\section{References}

1. Wang, H.; Naghavi, M.; Allen, C.; Barber, R.M.; Bhutta, Z.A.; Carter, A.; Casey, D.C.; Charlson, F.J.; Chen, A.Z.; Coates, M.M.; et al. Global, regional, and national life expectancy, all-cause mortality, and cause-specific mortality for 249 causes of death, 1980-2015: A systematic analysis for the Global Burden of Disease Study 2015. Lancet 2016, 388, 1459-1544. [CrossRef]

2. Monedero, V.; Collado, M.C.; Rodríguez-Díaz, J. Therapeutic opportunities in intestinal microbiota-virus interactions. Trends Biotechnol. 2018, 36, 645-648. [CrossRef] [PubMed]

3. Monedero, V.; Buesa, J.; Rodríguez-Díaz, J. The interactions between host glycobiology, bacterial microbiota, and viruses in the gut. Viruses 2018, 10, 96. [CrossRef] [PubMed]

4. Kim, A.H.J.; Hogarty, M.P.; Harris, V.C.; Baldridge, M.T. The complex interactions between rotavirus and the gut microbiota. Front. Cell. Infect. Microbiol. 2021, 10, 820. [CrossRef] [PubMed]

5. Roth, A.N.; Grau, K.R.; Karst, S.M. Diverse mechanisms underlie enhancement of enteric viruses by the mammalian intestinal microbiota. Viruses 2019, 11, 760. [CrossRef] [PubMed]

6. Harris, V.; Ali, A.; Fuentes, S.; Korpela, K.; Kazi, M.; Tate, J.; Parashar, U.; Wiersinga, W.J.; Giaquinto, C.; de Weerth, C.; et al. Rotavirus vaccine response correlates with the infant gut microbiota composition in Pakistan. Gut Microbes 2018, 9, 93-101. [CrossRef] [PubMed]

7. Harris, V.C.; Armah, G.; Fuentes, S.; Korpela, K.E.; Parashar, U.; Victor, J.C.; Tate, J.; de Weerth, C.; Giaquinto, C.; Wiersinga, W.J.; et al. Significant correlation between the infant gut microbiome and rotavirus vaccine response in rural Ghana. J. Infect. Dis. 2017, 215, 34-41. [CrossRef] [PubMed]

8. Uchiyama, R.; Chassaing, B.; Zhang, B.; Gewirtz, A.T. Antibiotic treatment suppresses rotavirus infection and enhances specific humoral immunity. J. Infect. Dis. 2014, 210, 171-182. [CrossRef] 
9. Gozalbo-Rovira, R.; Rubio-Del-campo, A.; Santiso-Bellón, C.; Vila-Vicent, S.; Buesa, J.; Delgado, S.; Molinero, N.; Margolles, A.; Yebra, M.J.; Collado, M.C.; et al. Interaction of intestinal bacteria with human rotavirus during infection in children. Int. J. Mol. Sci. 2021, 22, 1010. [CrossRef]

10. Goto, Y.; Uematsu, S.; Kiyono, H. Epithelial glycosylation in gut homeostasis and inflammation. Nat. Immunol. 2016, 17, 1244-1251. [CrossRef]

11. Gozalbo-Rovira, R.; Ciges-Tomas, J.R.; Vila-Vicent, S.; Buesa, J.; Santiso-Bellón, C.; Monedero, V.; Yebra, M.J.; Marina, A.; Rodríguez-Díaz, J. Unraveling the role of the secretor antigen in human rotavirus attachment to histo-blood group antigens. PLoS Pathog. 2019, 15, e1007865. [CrossRef] [PubMed]

12. Xu, S.; Ahmed, L.U.; Stuckert, M.R.; McGinnis, K.R.; Liu, Y.; Tan, M.; Huang, P.; Zhong, W.; Zhao, D.; Jiang, X.; et al. Molecular basis of P[II] major human rotavirus VP8* domain recognition of histo-blood group antigens. PLoS Pathog. 2020, 16, e1008386. [CrossRef] [PubMed]

13. Ramig, R.F. The effects of host age, virus dose, and virus strain on heterologous rotavirus infection of suckling mice. Microb. Pathog. 1988, 4, 189-202. [CrossRef]

14. Arnold, M.; Patton, J.T.; McDonald, S.M. Culturing, storage, and quantification of rotaviruses. Curr. Protoc. Microbiol. 2009, 15. [CrossRef]

15. Jothikumar, N.; Kang, G.; Hill, V.R. Broadly reactive TaqMan®assay for real-time RT-PCR detection of rotavirus in clinical and environmental samples. J. Virol. Methods 2009, 155, 126-131. [CrossRef]

16. Rakoff-Nahoum, S.; Paglino, J.; Eslami-Varzaneh, F.; Edberg, S.; Medzhitov, R. Recognition of commensal microflora by toll-like receptors is required for intestinal homeostasis. Cell 2004, 118, 229-241. [CrossRef] [PubMed]

17. Rubio-Del-Campo, A.; Gozalbo-Rovira, R.; Moya-Gonzálvez, E.M.; Alberola, J.; Rodríguez-Díaz, J.; Yebra, M.J. Infant gut microbiota modulation by human milk disaccharides in humanized microbiome mice. Gut Microbes 2021, 13, 1-20. [CrossRef]

18. Pfaffl, M.W.; Horgan, G.W.; Dempfle, L. Relative expression software tool (REST) for group-wise comparison and statistical analysis of relative expression results in real-time PCR. Nucleic Acids Res. 2002, 30, e36. [CrossRef]

19. Rubio-Del-Campo, A.; Alcántara, C.; Collado, M.C.; Rodríguez-Díaz, J.; Yebra, M.J. Human milk and mucosa-associated disaccharides impact on cultured infant fecal microbiota. Sci. Rep. 2020, 10, 1-12.

20. Caporaso, J.G.; Kuczynski, J.; Stombaugh, J.; Bittinger, K.; Bushman, F.D.; Costello, E.K.; Fierer, N.; Peña, A.G.; Goodrich, J.K.; Gordon, J.I.; et al. QIIME allows analysis of high-throughput community sequencing data. Nat. Methods 2010, 7, 335-336. [CrossRef]

21. Chong, J.; Liu, P.; Zhou, G.; Xia, J. Using MicrobiomeAnalyst for comprehensive statistical, functional, and meta-analysis of microbiome data. Nat. Protoc. 2020, 15, 799-821. [CrossRef]

22. Pérez-Ortín, R.; Vila-Vicent, S.; Carmona-Vicente, N.; Santiso-Bellón, C.; Rodríguez-Díaz, J.; Buesa, J. Histo-blood group antigens in children with symptomatic rotavirus infection. Viruses 2019, 11, 339. [CrossRef] [PubMed]

23. Witjes, V.M.; Boleij, A.; Halffman, W. Reducing versus embracing variation as strategies for reproducibility: The microbiome of laboratory mice. Animals 2020, 10, 2415. [CrossRef]

24. Schnepf, D.; Hernandez, P.; Mahlakõiv, T.; Crotta, S.; Sullender, M.E.; Peterson, S.T.; Ohnemus, A.; Michiels, C.; Gentle, I.; Dumoutier, L.; et al. Rotavirus susceptibility of antibiotic-treated mice ascribed to diminished expression of interleukin-22 2. bioRxiv 2021. [CrossRef]

25. Li, N.; Ma, W.T.; Pang, M.; Fan, Q.L.; Hua, J.L. The commensal microbiota and viral infection: A comprehensive review. Front. Immunol. 2019, 10, 1551. [CrossRef]

26. Abt, M.C.; Osborne, L.C.; Monticelli, L.A.; Doering, T.A.; Alenghat, T.; Sonnenberg, G.F.; Paley, M.A.; Antenus, M.; Williams, K.L.; Erikson, J.; et al. Commensal Bacteria Calibrate the Activation Threshold of Innate Antiviral Immunity. Immunity 2012, 37, 158-170. [CrossRef]

27. Stefan, K.L.; Kim, M.V.; Iwasaki, A.; Kasper, D.L. Commensal microbiota modulation of natural resistance to virus infection. Cell 2020, 183, 1312-1324. [CrossRef] [PubMed]

28. Yaron, J.R.; Ambadapadi, S.; Zhang, L.; Chavan, R.N.; Tibbetts, S.A.; Keinan, S.; Varsani, A.; Maldonado, J.; Kraberger, S.; Tafoya, A.M.; et al. Immune protection is dependent on the gut microbiome in a lethal mouse gammaherpesviral infection. Sci. Rep. 2020, 10, 1-13. [CrossRef] [PubMed]

29. Antunes, K.H.; Fachi, J.L.; de Paula, R.; da Silva, E.F.; Pral, L.P.; dos Santos, A.Á.; Dias, G.B.M.; Vargas, J.E.; Puga, R.; Mayer, F.Q.; et al. Microbiota-derived acetate protects against respiratory syncytial virus infection through a GPR43-type 1 interferon response. Nat. Commun. 2019, 10, 1-17. [CrossRef]

30. Yang, X.-L.; Wang, G.; Xie, J.-Y.; Li, H.; Liu, W.; Zhu, S.J. The intestinal microbiome primes host innate immunity against enteric virus 1 systemic infection through type I interferon 2. Res. Sq. 2021, 12, e00366-21.

31. Thackray, L.B.; Handley, S.A.; Gorman, M.J.; Poddar, S.; Bagadia, P.; Briseño, C.G.; Theisen, D.J.; Tan, Q.; Hykes, B.L.; Lin, H.; et al. Oral antibiotic treatment of mice exacerbates the disease severity of multiple flavivirus infections. Cell Rep. 2018, 22, 3440-3453. [CrossRef]

32. Kandasamy, S.; Vlasova, A.N.; Fischer, D.; Kumar, A.; Chattha, K.S.; Rauf, A.; Shao, L.; Langel, S.N.; Rajashekara, G.; Saif, L.J. Differential Effects of Escherichia coli Nissle and Lactobacillus rhamnosus Strain GG on Human Rotavirus Binding, Infection, and B Cell Immunity. J. Immunol. 2016, 196, 1780-1789. [CrossRef] 
33. Zhang, Z.; Xiang, Y.; Li, N.; Wang, B.; Ai, H.; Wang, X.; Huang, L.; Zheng, Y. Protective effects of Lactobacillus rhamnosus GG against human rotavirus-induced diarrhoea in a neonatal mouse model. Pathog. Dis. 2013, 67, 184-191. [CrossRef] [PubMed]

34. Jiang, B.; Snipes-Magaldi, L.; Dennehy, P.; Keyserling, H.; Holman, R.C.; Bresee, J.; Gentsch, J.; Glass, R.I. Cytokines as mediators for or effectors against rotavirus disease in children. Clin. Diagn. Lab. Immunol. 2003, 10, 995-1001. [CrossRef]

35. Kadooka, Y.; Tominari, K.; Sakai, F.; Yasui, H. Prevention of rotavirus-induced diarrhea by preferential secretion of IgA in breast milk via maternal administration of lactobacillus gasseri SBT2055. J. Pediatr. Gastroenterol. Nutr. 2012, 55, 66-71. [CrossRef] [PubMed]

36. Wen, K.; Azevedo, M.S.P.; Gonzalez, A.; Zhang, W.; Saif, L.J.; Li, G.; Yousef, A.; Yuan, L. Toll-like receptor and innate cytokine responses induced by lactobacilli colonization and human rotavirus infection in gnotobiotic pigs. Vet. Immunol. Immunopathol. 2009, 127, 304-315. [CrossRef] [PubMed]

37. Lin, B.; Saito, M.; Sakakibara, Y.; Hayashi, Y.; Yanagisawa, M.; Iwamori, M. Characterization of three members of murine $\alpha 1$, 2-fucosyltransferases: Change in the expression of the Se gene in the intestine of mice after administration of microbes. Arch. Biochem. Biophys. 2001, 388, 207-215. [CrossRef] [PubMed]

38. Guo, Y.; Candelero-Rueda, R.A.; Saif, L.J.; Vlasova, A.N. Infection of porcine small intestinal enteroids with human and pig rotavirus A strains reveals contrasting roles for histo-blood group antigens and terminal sialic acids. PLoS Pathog. 2021, 17, e1009237. [CrossRef] [PubMed]

39. Loy, A.; Pfann, C.; Steinberger, M.; Hanson, B.; Herp, S.; Brugiroux, S.; Gomes Neto, J.C.; Boekschoten, M.V.; Schwab, C.; Urich, T.; et al. Lifestyle and Horizontal Gene Transfer-Mediated Evolution of Mucispirillum schaedleri, a Core Member of the Murine Gut Microbiota. mSystems 2017, 2, e00171-16. [CrossRef]

40. Herp, S.; Brugiroux, S.; Garzetti, D.; Ring, D.; Jochum, L.M.; Beutler, M.; Eberl, C.; Hussain, S.; Walter, S.; Gerlach, R.G.; et al. Mucispirillum schaedleri Antagonizes Salmonella Virulence to Protect Mice against Colitis. Cell Host Microbe 2019, 25, 681-694. [CrossRef]

41. Konikoff, T.; Gophna, U. Oscillospira: A Central, Enigmatic Component of the Human Gut Microbiota. Trends Microbiol. 2016, 24, 523-524. [CrossRef]

42. Natividad, J.M.; Lamas, B.; Pham, H.P.; Michel, M.L.; Rainteau, D.; Bridonneau, C.; Da Costa, G.; Van Hylckama Vlieg, J.; Sovran, B.; Chamignon, C.; et al. Bilophila wadsworthia aggravates high fat diet induced metabolic dysfunctions in mice. Nat. Commun. 2018, 9, 1-15. [CrossRef] [PubMed]

43. Di, J.B.; Gai, Z.T. Protective efficacy of probiotics on the treatment of acute rotavirus diarrhea in children: An updated metaanalysis. Eur. Rev. Med. Pharmacol. Sci. 2020, 24, 9675-9683. [PubMed]

44. Thompson, A.; Van Moorlehem, E.; Aich, P. Probiotic-induced priming of innate immunity to protect against rotaviral infection. Probiotics Antimicrob. Proteins 2010, 2, 90-97. [CrossRef] [PubMed]

45. Vlasova, A.N.; Chattha, K.S.; Kandasamy, S.; Liu, Z.; Esseili, M.; Shao, L.; Rajashekara, G.; Saif, L.J. Lactobacilli and bifidobacteria promote immune homeostasis by modulating innate immune responses to human rotavirus in neonatal gnotobiotic pigs. PLoS ONE 2013, 8, e76962. [CrossRef] [PubMed]

46. Shi, Z.; Zou, J.; Zhang, Z.; Zhao, X.; Noriega, J.; Zhang, B.; Zhao, C.; Ingle, H.; Bittinger, K.; Mattei, L.M.; et al. Segmented filamentous bacteria prevent and cure rotavirus infection. Cell 2019, 179, 644-658. [CrossRef] [PubMed]

47. Rodriguez-Diaz, J.; Garcia-Mantrana, I.; Vila-Vicent, S.; Gozalbo-Rovira, R.; Buesa, J.; Monedero, V.; Collado, M.C. Relevance of secretor status genotype and microbiota composition in susceptibility to rotavirus and norovirus infections in humans. Sci. Rep. 2017, 7, 1-10.

48. Kawahara, T.; Makizaki, Y.; Oikawa, Y.; Tanaka, Y.; Maeda, A.; Shimakawa, M.; Komoto, S.; Moriguchi, K.; Ohno, H.; Taniguchi, K. Oral administration of Bifidobacterium bifidum G9-1 alleviates rotavirus gastroenteritis through regulation of intestinal homeostasis by inducing mucosal protective factors. PLoS ONE 2017, 12, e0173979. [CrossRef]

49. Salminen, S.; Nybom, S.; Meriluoto, J.; Collado, M.C.; Vesterlund, S.; El-Nezami, H. Interaction of probiotics and pathogensBenefits to human health? Curr. Opin. Biotechnol. 2010, 21, 157-167. [CrossRef]

50. Almand, E.A.; Moore, M.D.; Outlaw, J.; Jaykus, L.-A. Human norovirus binding to select bacteria representative of the human gut microbiota. PLoS ONE 2017, 12, e0173124. [CrossRef]

51. Jones, M.K.; Watanabe, M.; Zhu, S.; Graves, C.L.; Keyes, L.R.; Grau, K.R.; Gonzalez-Hernandez, M.B.; Iovine, N.M.; Wobus, C.E.; Vinje, J.; et al. Enteric bacteria promote human and mouse norovirus infection of B cells. Science 2014, 346, 755-759. [CrossRef] [PubMed]

52. Kuss, S.K.; Best, G.T.; Etheredge, C.A.; Pruijssers, A.J.; Frierson, J.M.; Hooper, L.V.; Dermody, T.S.; Pfeiffer, J.K. Intestinal microbiota promote enteric virus replication and systemic pathogenesis. Science 2011, 334, 249-252. [CrossRef] [PubMed]

53. Li, D.; Breiman, A.; le Pendu, J.; Uyttendaele, M. Binding to histo-blood group antigen-expressing bacteria protects human norovirus from acute heat stress. Front. Microbiol. 2015, 6, 659. [CrossRef] [PubMed] 